\title{
PALINOLOGIA DOS CARVÕES PALEOZÓICOS DO ESTADO DE SÃO PAULO, BRASIL. I - O CARVÃO DE BURI
}

\begin{abstract}
RESUMO
São inúmeros os problemas relacionados à estratigrafia do Subgrupo Itararé, unidade representativa da sedimentação glacial paleozóica da Bacia do Paraná. Tais dificuldades são decorrentes do fato de a seqüência em questão constituir um conjunto de grande diversidade litológica e variação faciológica, no qual os fósseis são relativamente raros. Dentre os diversos termos litológicos presentes no Subgrupo Itararé, destacam-se os carvões pela potencialidade bioestratigráfica. Conhecidos há longo tempo, representam pelo menos três ocorrências: Buri, Monte Mor e Cerquilho. $\mathrm{O}$ trabalho ora apresentado refere-se especificamente aos resultados palinológicos já obtidos a partir do estudo de amostras do Carvão de Buri, que revelam ser ele o mais antigo entre os três níveis estudados. Trinta e quatro taxa foram identificados nas associações, cujo predomínio absoluto é de esporos triletes zonados. Diversas espécies presentes permitem sua correlação com sedimentos da Bacia de Paganzo (Formação Malanzán - Membro Estratos Carbonosos), de idade carbonífera, situada provavelmente no Westfaliano, em condições de deposição continentais.
\end{abstract}

\begin{abstract}
Numerous stratigraphic problems persist regarding the Itarare Subgroup, the principal unit representative of Late Paleozoic glaciation in the Paraná Basin, as the result of the great lithologic and faciologic variety and the relative rarity of fossils in this sequence. Thus, because of their potential biostratigraphic significance, special attention is due to the classic coal occurrences at Buri, Monte Mor and Cerquilho in the State of São Paulo. Palynological work presented here reveals that the Buri coal is the oldest of these three levels. Thirty-four taxa have been identified in the assemblage, with an absolute predominance of zonate spores. Various species allow correlation with Carboniferous sediments of the Paganzo Basin, Argentina (Malanzán Formation - Estratos Carbonosos Member), of probable Westphalian age, deposited in a deltaic environment.
\end{abstract}

\section{INTRODUÇÃO}

Representando ambientes restritos e relativamente raros, os depósitos de carvão, pela facilidade de preservação da matéria orgânica e conseqüentemente de fósseis, encerram grande potencialidade no que se refere a reconstituições da história geológica de suas deposições.

As ocorrências presentes no Estado de São Paulo, apesar de pouco expressivas em termos geográficos, vêm, por esta razão, há muito tempo despertando a atenção dos especialistas. Ressalta-se ainda que estes carvões ocorrem em seqüência estratigráfica de grande complexidade faciológica e em geral pouco fossilífera, o que certamente contribui para aumentar a sua importância nos estudos geológicos regionais.

Dentre os diversos grupos de fósseis presentes nos carvões destacam-se os palinomorfos pela possibilidade de assinalar com precisão a idade desses eventos, bem como sua correlação estratigráfica com níveis cronologicamente equivalentes, além de interpretações paleoambientais, paleogeográficas, paleoecológicas e paleoclimáticas. Embora existam dados relativos ao estudo destes microfósseis para todos os carvões presentes no Estado, o nível de abordagem adotado até agora pode ser considerado demasiadamente superficial, dificultando a obtenção de conclusões satisfatórias. Por esta razão, o trabalho em questão tem como objetivo retomar o tema, reestudando as três ocorrências principais conhecidas: os carvões de Buri, Monte Mor e Cerquilho. O primeiro escolhido para fins de publicações foi o de Buri, em função dos resultados obtidos, que, contrariamente ao que registra a literatura consultada, foi considerado o mais antigo dos três. 


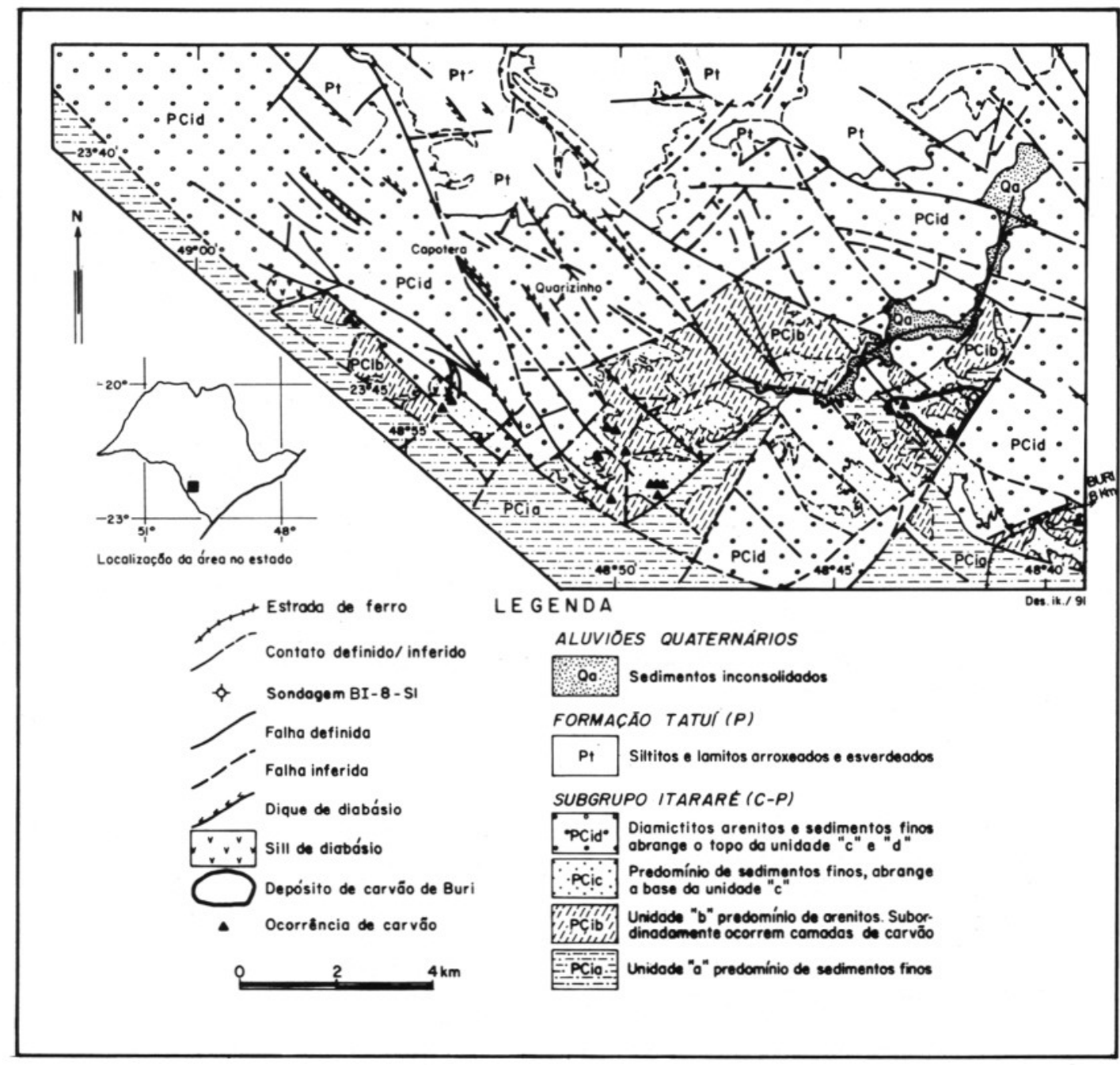

FIGURA 1 - Mapa geológico da área (adaptado de CABRAL JÚNIOR \& MOTTA, 1985).

\section{CONSIDERAÇÕES GERAIS}

\subsection{Geologia geral}

A região onde aflora o Carvão de Buri (Paineira e outros níveis carbonosos associados) situa-se no flanco leste da Bacia do Paraná, sul do Estado de São Paulo, nas proximidades dos municípios de Buri e Itapeva. É geologicamente representada pelos sedimentos permocarboníferos do Subgrupo Itararé e Formação Tatuí, conforme pode ser visualizado na figura 1 . Do ponto de vista tectônico, a área exibe um sistema de falhas escalonadas do tipo "horst" e "graben", com duas direções preferenciais (NW-SE e NE$\mathrm{SW})$, cujos rejeitos variam até mais de duas centenas de metros (CABRAL JÚNIOR \& MOTTA, 1985). É marcante ainda a presença de diques de diabásio com tais orientações, notadamente a direção noroeste, muitas vezes ocupando os planos de falhas. Secundariamente, observa-se a presença de sills.

As camadas de carvão, bem como as litologias a elas associadas, estão posicionadas no Subgrupo Itararé. $\mathrm{Na}$ área, tanto em superfície como em subsuperfície, esta unidade caracteriza-se por apresentar uma grande diversidade litológi$\mathrm{ca}$, onde se destacam arenitos de granulometria variada, desde muito finos a conglomeráticos, diamictitos, ritmitos, siltitos, folhelhos, argilitos e os níveis de carvão (CABRAL JÚNIOR \& MOTTA, op. cit.). Tais litologias foram agrupadas por esses autores em quatro conjuntos faciológicos informais, representando cada um deles províncias fisiográficas distintas. Assim, da ba- 
se para o topo, têm-se as unidades "a", "b", "c" e "d", sendo que níveis carbonosos podem ser encontrados nas unidades "b" e "c". O estudo em questão refere-se aos depósitos pertencentes à unidade "b", que é o principal nível carbonoso da região de Buri-Itapeva.

A figura 2 exibe o empilhamento sedimentar de fácies para as unidades "a" e "b" de Buri, segundo SOUZA-FILHO (1986). Conforme pode ser observado nesta figura, a unidade "a" constitui-se predominantemente por pelitos e, em segundo plano, por arenitos. Os primeiros correspondem a siltitos de cores claras a acinzentadas ou esverdeadas, maciços ou com estratificações onduladas, paralelas ou microcruzadas, rompidas ou não por organismos. Secundariamente, verificam-se concreções piritosas e estruturas deposicionais de fluxo. Os arenitos associados são muito finos, limpos, de cores esbranquiçadas e pouco micáceos. Ocorrem em corpos estruturados com estratificações cruzadas de baixo ângulo, estratificação microcruzada e laminações onduladas.

A unidade "b", por sua vez, compõe-se essencialmente por arenitos finos a médios, subordinadamente conglomeráticos, siltitos e camadas de carvão. Os arenitos apresentam granodecrescência ascendente, são regularmente selecionados, com pouca matriz e freqüentes fragmentos carbonosos. Na base, são comuns estruturas acanaladas, onde predominam galhas de argila, níveis conglomeráticos e conglomerados polimíticos. As estruturas sedimentares presentes resumem-se às estratificações cruzadas. Estas variam de grande a médio porte, principalmente na base, diminuindo em direção ao topo, onde passa a ser do tipo microcruzada. Subordinadamente, é comum a presença de bioturbação provocada por raízes.

O conjunto faciológico detectado, bem como o empilhamento sedimentar reconhecido, refletem uma situação fisiográfica dominada por sistema deltaico, em mares rasos plataformais. Neste contexto, a unidade "a" representa o domínio prodeltaico, que gradativamente passa para os domínios de frente e planície de inundação. De acordo com CABRAL JÚNIOR \& MOTTA (op. cit.), os carvões foram depositados neste último ambiente (unidade "b").

\subsection{Paleontologia}

Os fósseis mais comuns nos carvões paleozóicos do Estado de São Paulo são sem dúvida os vegetais. Com relação ao estudo do seu documentário macroscópico, pontificam os trabalhos do pesquisador J. H. MILLAN, que têm permitido a obtenção de conclusões relativamente
DESCRIĢĀO FACIOLÓGICA

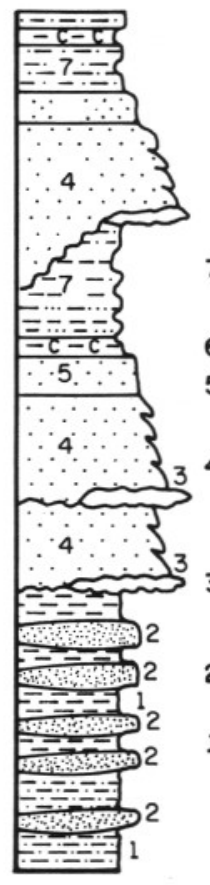

INTERPRETAÇ̄̄O

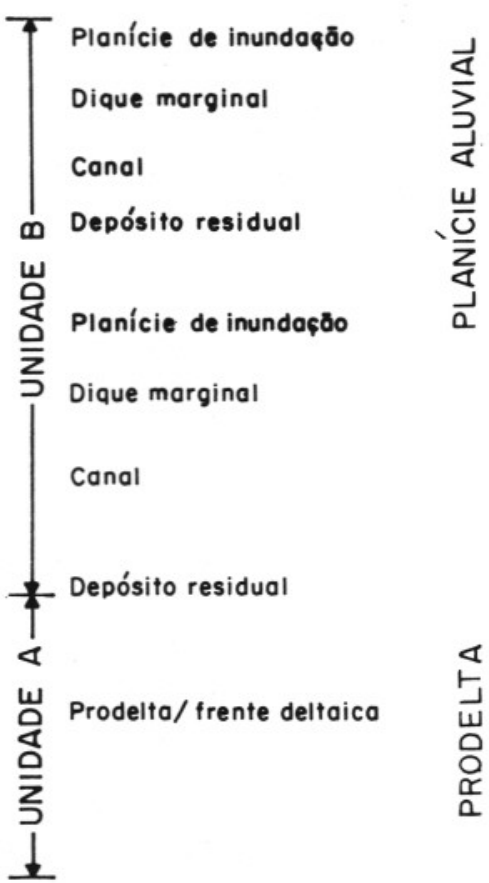

FIGURA 2 - Seqüência ideal de fácies para as unidades A e B de Buri (adaptada de SOUZA FILHO, 1986). 
surpreendentes a respeito da idade dos níveis estudados. RÖSLER \& PERINOTTO (1985) noticiaram a ocorrência de uma impressão carbonosa representando parte de uma pina com várias pínulas, atribuída ao gênero Nothorhacopteris sp. Este referido exemplar de megarresto vegetal foi registrado no mesmo nível de siltito carbonoso do poço BI-8-SP, também tratado neste trabalho.

Vale mencionar que, dos três níveis de carvão citados, é o de Buri o menos conhecido, já que a maior parte da literatura existente (exceção feita aos trabalhos de N. M. TRINDADE e colaboradores) é concernente aos carvões de Monte Mor e Cerquilho.

\section{RESULTADOS PALINOLÓGICOS}

\subsection{Considerações prévias}

Os estudos palinológicos dos carvões paleozóicos do Estado de São Paulo foram iniciados na década de 50. O grupo dos megásporos foi o primeiro a ser enfocado, fornecendo como resultado expressiva bibliografia, na qual se destacam os trabalhos de TRINDADE (1959, 1960, 1970), SOMMER \& TRINDADE (1965), TRINDADE \& SOMMER (1966) e FERREIRA \& SOMMER (1982). Estes estudos já sugerem a possibilidade de uma idade carbonífera ao invés de permiana para os níveis estudados.

Com relação aos micrósporos (lato sensu) o enfoque é muito mais recente, tendo sido iniciado por DAEMON (1974), representando o trabalho desenvolvido pela Petrobrás. No âmbito da pesquisa acadêmica destacam-se aqui as contribuições de SAAD (1977), ARAI (1980) e SUNDARAM $(1980,1987)$. Todos estes trabalhos são em verdade de temática bastante ampla, incluindo dados relativos aos carvões, mas não se direcionando especificamente a eles.

\subsection{Materiais e métodos}

Foram tomadas para estudo duas amostras litologicamente distintas. A primeira é um carvão proveniente do rejeito da antiga mina do município de Buri, coletada e cedida para estudo pelos Profs. Antonio Roberto Saad e Paulo Roberto dos Santos e pelo geólogo Marsis Cabral Júnior. A outra corresponde a um siltito carbonoso cinza, à profundidade de $64,3 \mathrm{~m}$ do poço BI-8-SP, realizado pelo Instituto de Pesquisas Tecnológicas do Estado de São Paulo.

As amostras foram submetidas a tratamento palinológico habitual, que consiste na dissolução completa de seus componentes minerais, clareamento da matéria orgânica e concentração final do resíduo.

Um total de oito lâminas foi montado, sendo cinco relativas à amostra de carvão e três ao siltito carbonoso. As lâminas foram estudadas qualitativa e quantitativamente no fotomicroscópio Zeiss modelo Ortholux, pertencente ao Departamento de Paleontologia e Estratigrafia do IGc-USP. Para execução das fotomicrografias foi utilizado o equipamento de câmara modelo Olympus Vanox pertencente ao Prof. Dr. Jean Pierre Ybert. As lâminas encontram-se depositadas na coleção científica (palinoteca) do DPEIGc/USP sob os números GP/4T-299 a GP/4T-303 (carvão) e GP/4T-304 a GP/4T-306 (siltito carbonoso).

\subsection{Análise qualitativa}

Trinta e quatro taxa, correspondentes a esporos e grãos de pólen, representando vinte e dois gêneros, foram identificados nas associações microflorísticas de Buri. Para isto foram consultados, entre outros, os trabalhos de ARCHANGELSKY \& GAMERRO (1979), AZCUY (1975ab), BOSE \& KAR (1966), BURJACK (1978), CAUDURO (1970), FABRICIO (1981), FOSTER (1979), HART (1965), MAHESHWARI (1967, 1969), MARQUES-TOIGO (1973), MARQUES-TOIGO \& PONS (1976), MENENDEZ \& AZCUY (1972), PONS (1975) e YBERT (1975). A relação completa, acompanhada das descrições das formas encontradas, é a seguinte:

\section{SPORITES}

Anteturma Proximegerminantes POTONIÉ, 1950.

Turma Triletes (REINSCH, 1881) DETTMANN, 1963.

Suprasubturma Acavatitriletes DETTMANN, 1963.

Subturma Azonotriletes (LUBER, 1935) DETTMANN, 1963.

Infraturma Laevigati (BENNIE \& KIDSTON, 1886) POTONIÉ, 1956.

\section{Gênero Punctatisporites (IBRAHIM, 1933) POTONIÉ \& KREMP, 1954.}

Punctatisporites gretensis BALME \& HENNELLY, 1956.

(Est. I fig. 1)

Punctatisporites gretensis (BALME \& HENNELLY, 1956) forma minor HART, 1965.

(Est. I fig. 2) 
Punctatisporites foveolatus MAHESWARI, 1969.

(Est. I fig. 3)

Gênero Dictyophyllidites (COUPER, 1958) DETTMANN, 1963.

Dictyophyllidites sp. 1.

(Est. II fig. 3)

Dictyophyllidites sp. 2.

(Est. II. fig. 4)

Infraturma Apiculati (BENNIE \& KIDSTON, 1886) POTONIÉ, 1965.

Subinfraturma Nodati DYBOWA \& JACHOWICZ, 1957.

Gênero Apiculiretusispora STREEL, 1964.

Apiculiretusispora sp. cf. A. tuberculata AZCUY, 1975.

(Est. I fig. 5)

Gênero Apiculatisporis (IBRAHIM, 1933) POTONIÉ \& KREMP, 1956.

Apiculatisporis $\mathrm{sp}$.

(Est. I fig. 6)

Subinfraturma Granulati DYBOWA \& JACHOWICZ, 1957.

Gênero Granulatisporites IBRAHIM, 1933.

Granulatisporites sp. cf. G. varigranifer MENENDEZ \& AZCUY, 1971.

(Est. I fig. 4)

Subinfraturma Baculati DYBOWA \& JACHOWICZ, 1957.

Gênero Raistrickia (SCHOPF, WILSON \& BENTALL, 1944) POTONIÉ \& KREMP, 1954.

Raistrickia paganciana AZCUY, 1975.

(Est. 1 fig. 13)

Raistrickia sp. cf. $R$. paganciana AZCUY, 1975.

(Est. I fig. 14)

Raistrickia rotunda AZCUY, 1975.

(Est. I figs. 10 e 12)

Raistrickia sp. 1.

(Est. I fig. 11)

Raistrickia sp. 2.

(Est. II fig. 1)

Infraturma Murornati POTONIÉ \& KREMP, 1954.

Gênero Convolutispora HOFFMEISTER, STAPLIN \& MALLOY, 1955.

Convolutispora $\mathrm{sp}$.

(Est. I fig. 7)

Gênero Secarisporites NEVES, 1961.

Secarisporites irregularis AZCUY, 1975.

(Est. I fig. 9)

Secarisporites $\mathrm{sp}$.

(Est. I fig. 8)

Gênero Foveosporites BALME, 1957.

Foveosporites hortonensis (PLAYFORD, 1964) AZCUY, 1975.

(Est. II fig 2.)

Supra-subturma Laminatitriletes SMITH \& BUTTERWORTH, 1967.

Subturma Zonolaminatitriletes SMITH \& BUTTERWORTH, 1967. 
Infraturma Cingulati (POTONIÉ \& KLAUS, 1954) DETTMANN, 1963.

Gênero Stenozonotriletes (NAUMOVA, 1939) HACQUEBARD, 1957.

Stenozonotriletes clarus ISHCHENKO, 1958.

(Est. II fig. 5)

Gênero Lundbladispora (BALME, 1963) PLAYFORD, 1965.

Lundbladispora braziliensis (PANT \& SRIVASTAVA, 1965) MARQUES-TOIGO \& PONS, 1976.

(Est. III fig. 4)

Gênero Murospora SOMERS, 1975.

Murospora bicingulata YBERT, 1975.

(Est. II fig. 6)

Infraturma Cingulicavati SMITH \& BUTTERWORTH, 1967.

Gênero Densosporites (BERRY, 1937) BUTTERWORTH, JANSONIUS, SMITH \& STAPLIN, 1964. Densosporites sp.

(Est. II fig. 7)

Gênero Cristatisporites (POTONIÉ \& KREMP, 1954) BUTTERWORTH et al., 1964.

Cristatisporites spinosus (MENENDEZ \& AZCUY, 1972) PLAYFORD 1978 (= Ancistrospora spinosa MENENDEZ \& AZCUY, 1972) (Est. II fig. 11)

Cristatisporites $\mathrm{cf}$. C. spinosus

(Est. II fig. 10)

Cristatisporites inordinatus (MENENDEZ \& AZCUY, 1972) PLAYFORD 1978 (= Ancistrospora inordinata MENENDEZ \& AZCUY, 1972)

(Est. II fig. 9)

Cristatisporites menendezii (MENENDEZ \& AZCUY, 1972) PLAYFORD 1978 (= Ancistrospora cf. A. verrucosa MENENDEZ \& AZCUY, 1972)

(Est. II fig. 12)

Cristatisporites sp. 1.

(Est. II fig. 8)

Cristatisporites sp 2.

(Est. II fig. 13)

Gênero Vallatisporites HACQUEBARD, 1957.

Vallatisporites vallatus HACQUEBARD, 1957.

(Est. III fig. 2)

Vallatisporites spinosus CAUDURO, 1970.

(Est. III fig. 3)

Vallatisporites sp. cf. V. ciliaris (LUBER, 1938) SULLIVAN, 1964.

(Est. III fig. 1)

Turma Monoletes IBRAHIM, 1933.

Subturma Azonomonoletes LUBER, 1955.

Infraturma Laevigatomonoletes DYBOWA \& JACHOWICZ, 1957.

Gênero Laevigatosporites IBRAHIM, 1933.

Laevigatosporites $\mathrm{sp}$.

(não ilustrado) 
Subturma Monosaccites (CHITALEY, 1951) POTONIÉ \& KREMP, 1954. Infraturma Aletesacciti LESCHICK, 1955.

Gênero Florinites SCHOPF, WILSON \& BENTALL, 1944.

Florinites sp.

(Est. III fig. 5)

Infraturma Apertacolpiti LELE, 1964.

Gênero Plicatipollenites LELE, 1964.

Plicatipollenites indicus LELE, 1964.

(Est. III fig. 6)

Plicatipollenites trigonalis LELE, 1964.

(Est. III fig. 9)

Infraturma Parasacciti MAHESHWARI, 1967.

Subinfraturma Divarisacciti BOSE \& KAR, 1966.

Gênero Divarisaccus VENKATACHALA \& KAR, 1965.

Divarisaccus sp.

(Est. III fig. 7)

Infraturma Vesiculomonoraditi (PANT, 1954) BRARADWAJ, 1955.

Gênero Potonieisporites LELE, 1964.

Potonieisporites brasiliensis (NAHUYS, ALPERN \& YBERT, 1968)

ARCHANGELSKY \& GAMERRO, 1979.

(Est. III fig. 8)

Subturma Disaccites COOKSON, 1947.

Infraturma Striatiti PANT, 1954.

Gênero Protohaploxypinus (SAMOILOVICH, 1953) HART, 1964.

Protohaploxypinus sp.

(Est. III fig. 10)

INCERTAE SEDIS

Grupo Acritarcha EVITT, 1963.

Subgrupo Acanthomorphitae (DOWNIE, EVITT \& SARJEANT, 1963) LISTER, 1970.

Gênero Micrhystridium (DEFLANDRE, 1937) STAPLIN, 1961.

Micrhystridium sp.

\subsection{Análise quantitativa}

O resultado da análise quantitativa dos grupos morfológicos foi obtido a partir da contagem de cem grãos para cada lâmina proveniente do carvão e cinqüenta grãos para cada lâmina proveniente do siltito carbonoso de subsuperfície. Os grupos de forma analisados foram os seguintes: (1) triletes lisos, (2) triletes ornamentados, (3) monoletes, (4) monossacados, (5) bissacados lisos e (6) bissacados estriados. Suas freqüências encontram-se ilustradas nos gráficos da figura 3 .
Dentre os triletes lisos, as espécies de maior expressão são as pertencentes ao gênero Punctatisporites. No que se refere aos triletes ornamentados (incluem os apiculados, zonados, cingulados e murornados), os resultados são relativamente semelhantes para as duas amostras, representados predominantemente pelos gêneros Vallatisporites e Lundbladispora, enquanto que os gêneros Foveosporites, Raistrickia e Ancistrospora são expressivos apenas nas lâminas da amostra de carvão. Esporos monoletes só foram 
verificados nas lâminas da amostra de carvão e são referíveis ao gênero Laevigatosporites. Os grãos de pólen monossacados têm maior freqüência nas lâminas da amostra de subsuperfície (siltito carbonoso) e são representados predominantemente pelos gêneros Potonieisporites e Plicatipollenites. Quanto aos grãos de pólen estriados, estes são representados por apenas um espécime, atribuído ao gênero Protohaploxypinus.

\section{DISCUSSÃO DOS RESULTADOS}

\subsection{Zoneamentos e correlação bioestratigráfica}

DAEMON \& QUADROS (1970) foram os primeiros autores a apresentar um zoneamento bioestratigráfico válido teoricamente para a seção neopaleozóica da Bacia do Paraná. Neste trabalho, o subgrupo Itararé, unidade na qual se enquadra o Carvão de Buri, é representado, da base para o topo, por três intervalos, designados como G, $\mathrm{H}$ e I. O segundo intervalo é dividido em três subintervalos $\left(\mathrm{H}_{1}, \mathrm{H}_{2}\right.$ e $\left.\mathrm{H}_{3}\right)$ e o terceiro em quatro $\left(I_{1}, I_{2}, I_{3}\right.$ e $\left.I_{4}\right)$. O esquema bioestratigráfico foi baseado apenas em grãos de pólen, sendo desconsiderados os esporos. Isto faz com que a simples presença de bissacados estriados, mesmo se primitivos ou raros, já seja suficiente para enquadrar as associações em intervalos pós/G-H

AZCUY (1976) e AZCUY \& JELIN (1980) estabeleceram um zoneamento bioestratigráfico para a seção neopaleozóica da vizinha Bacia de Paganzo. Neste estudo, fica evidente a existência de uma palinozona até então desconhecida para sedimentos gondvânicos, mais antiga que o invervalo $\mathrm{G}_{-} \mathrm{H}_{1}$ de DAEMON \& QUADROS (1970). Esta, aparentemente ausente na Bacia do Paraná, é, ao contrário das demais, caracteriza- da por esporos, notadamente do gênero Ancistrospora. Este taxon foi considerado como sinonímia e relocado ao gênero Cristatisporites por PLAYFORD (1978), colocação aceita por OTTONE \& AZCUY (1990). No entanto, é referido nesta discussão como no original, devido ao seu valor palinoestratigráfico consagrado.

SUNDARAM (1980) foi o primeiro autor a admitir, embora sem evidências conclusivas, a possibilidade da existência de níveis equivalentes às palinozonas mais basais da Bacia de $\mathrm{Pa}$ ganzo na Bacia do Paraná. O autor, em estudo que incluiu amostra da região de Buri, agrupou os palinomorfos identificados em duas zonas microflorísticas, designadas informalmente de $\mathrm{Pa}$ linozonas " $A$ " $e$ " $B$ ". A mais antiga, e que teria correspondência com a Palinozona I de AZCUY (1976), é caracterizada pelo domínio de esporos triletes zonados e cingulados (70 a $80 \%$ ). Subordinadamente, o autor mencionou a presença de grãos de pólen monossacados (cerca de $20 \%$ ) e uma baixa freqüência de dissacados. É importante notar que esta correlação foi efetuada com base em freqüências de grupos taxonômicos superiores a gênero, sendo que nenhum gênero típico da Palinozona I de AZCUY (1976) foi reconhecido.

ARAI (1980) foi outro autor a divulgar dados palinológicos sucintos relativos ao Carvão de Buri. Esse trabalho, bastante abrangente, foi elaborado com base no estudo de 166 amostras, fornecendo um esquema bioestratigráfico informal alternativo ao de DAEMON \& QUADROS (1970). Foram reconhecidas seis "fases" evolutivas, em função da presença e percentagem de grãos de pólen estriados. A "fase" mais antiga, denominada "Pré-Striatiti", é caracterizada pela ausência deste grupo e inclui a amostra proce-

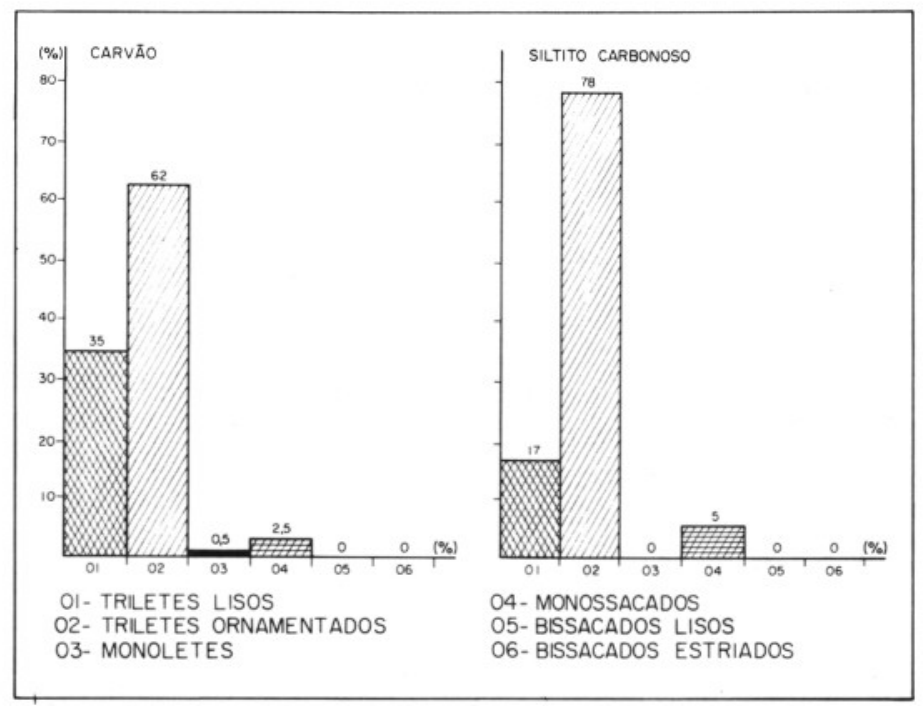

FIGURA 3 - Distribução quantitativa dos principais grupos estudados. 
dente de Buri. Nela, ARAI (op. cit.) identificou vários gêneros de esporos e grãos de pólen monossacados.

LIMA et al. (1983) foram os primeiros autores a reconhecer a presença de elementos típicos da Palinozona I de AZCUY (1976) e Palinozona de Ancistrospora de AZCUY \& JELIN (1980) na Bacia do Paraná. Os autores designaram informalmente o intervalo, caracterizado pela presença do gênero Ancistrospora, como "Pré-G", ressaltando o fato de que ele era mais primitivo que o intervalo basal $\mathrm{G}$ do esquema de DAEMON \& QUADROS (1970). A associação, proveniente da região de Araçoiaba da Serra, no Estado de São Paulo, conta com a presença da espécie Ancistrospora inordinata e com percentagens de esporos de cerca de $73 \%$. Grãos monossacados perfazem $25 \%$ do espectro polínico, que conta com cerca de $2 \%$ de dissacados estriados. Pelas características apresentadas, transicionais entre as Palinozonas I e II (esta última apresenta 5\% de grãos de pólen dissacados estriados) do esquema proposto para a Bacia de Paganzo, LIMA et al. (1983) optaram por posicionar a associação na faixa transicional entre as duas.

Qualitativamente, a assembléia ora detectada no Carvão de Buri enquadra-se perfeitamente dentro da Palinozona de Ancistrospora de AZCUY \& JELIN (1980). Nela, é expressivo o domínio dos esporos (cerca de 93\%). Várias das formas características desta palinozona estão presentes, destacando-se os gêneros Ancistrospora, Granulatisporites, Convolutispora, Raistrickia, Retusotriletes, Verrucosisporites, Cristatisporites e Punctatisportes. Por outro lado, a presença de grãos de pólen monossacados dos gêneros Florinites, Potonieisporites e Divarisaccus, bem como de um exemplar de dissacado estriado (Protohaploxypinus), poderia significar um caráter relativamente mais jovem para esta associação. Vale, contudo, mencionar que o conjunto distancia-se bastante, tanto qualitativa como quantitativamente, da Palinozona de Potonieisporites (AZCUY \& JELIN, 1980) da Bacia de Paganzo, na qual o gênero Ancistrospora é ausente, onde aumenta a representatividade dos grãos de pólen monossacados (Plicatipollenites indicus, Florinites verrucossus) e os bissacados perfazem cerca de $5 \%$. Por esta razão, a assembléia aqui estudada deve, tal como a de Araçoiaba da Serra, estudada por LIMA et al. (1983), ser enquadrada no limite entre as Palinozonas de Ancistrospora e Palinozona de Potonieisporites, embora as características gerais sejam sugestivas de que ela é ainda um pouco mais primitiva que essa última. Ainda com base na freqüência dos grupos de monossacados e estriados, a assembléia de Araçoiaba da Serra perfaz 25 e $2 \%$, respectivamente. Isto faz com que a assembléia, presente no Carvão de Buri, seja, até o momento, a mais antiga associação polínica conhecida no Neopaleozóico da Bacia do Paraná.

\subsection{Idade}

Uma idade carbonífera para o Carvão de Buri já vem sendo preconizada desde os estudos efetuados com base em megásporos, e é sustentada por estudos palinológicos efetuados posteriormente por SUNDARAM (1980 e 1987) e ARAI (1980). Contudo, outros autores, como CABRAL JÚNIOR \& MOTTA (1985), também baseados em informações palinológicas, posicionam a ocorrência no intervalo $\mathrm{H}_{1}$ de DAEMON \& QUADROS (1970), que representaria, em termos aproximativos, o Permiano Inferior (Sakmariano). SAAD (1977), também com base em grãos de pólen e esporos, posicionou a ocorrência no intervalo $\mathrm{H}_{2}$ do mesmo esquema, o que conduziria também a uma idade permiana.

Os dados ora divulgados, ao permitirem o posicionamento da ocorrência no intervalo informal "Pré-G", possibilitam a confirmação de idade carbonífera para o Carvão de Buri. Uma idade mais antiga que a westfaliana deve ser descartada, pela ocorrência, ainda que rara, do gênero Protohaploxypinus. Por outro lado, uma idade mais nova também pode ser eliminada, já que se refere ao intervalo bioestratigráfico $\mathrm{G}-\mathrm{H}_{1}$ imediatamente superior, com o qual não se pode estabelecer uma adequada correlação. Logo, é no Westfaliano que devem ser posicionados os níveis portadores do Carvão de Buri.

Resultados concernentes ao Carvão de Monte Mor, que não corroboram a proposição de MILLAN (1987), foram divulgados preliminarmente por SOUZA et al. (1993) e demonstram ser este mais jovem que o Carvão de Buri.

Conseqüentemente, é o Carvão de Buri o mais antigo dos níveis de carvão do Estado de São Paulo, justificando assim sua escolha para divulgação dos dados palinológicos a ele relativos.

\subsection{Correlação estratigráfica}

A ausência de níveis-guia regionais no Subgrupo Itararé, aliada ao fato da não utilização sistemática de análises palinológicas nos estudos relativos a esta unidade, têm dificultado por vezes estabelecer o posicionamento estratigráfico correto do intervalo analisado. Em decorrência, o entendimento do seu contexto paleogeográfico no âmbito da Bacia do Paraná é grandemente prejudicado.

Outro fator importante nesta questão diz respeito à tectônica pós-Itararé, notadamente a mesocenozóica. Como se sabe, a reativação sulatlantiana provocou, entre outras coisas, falha- 


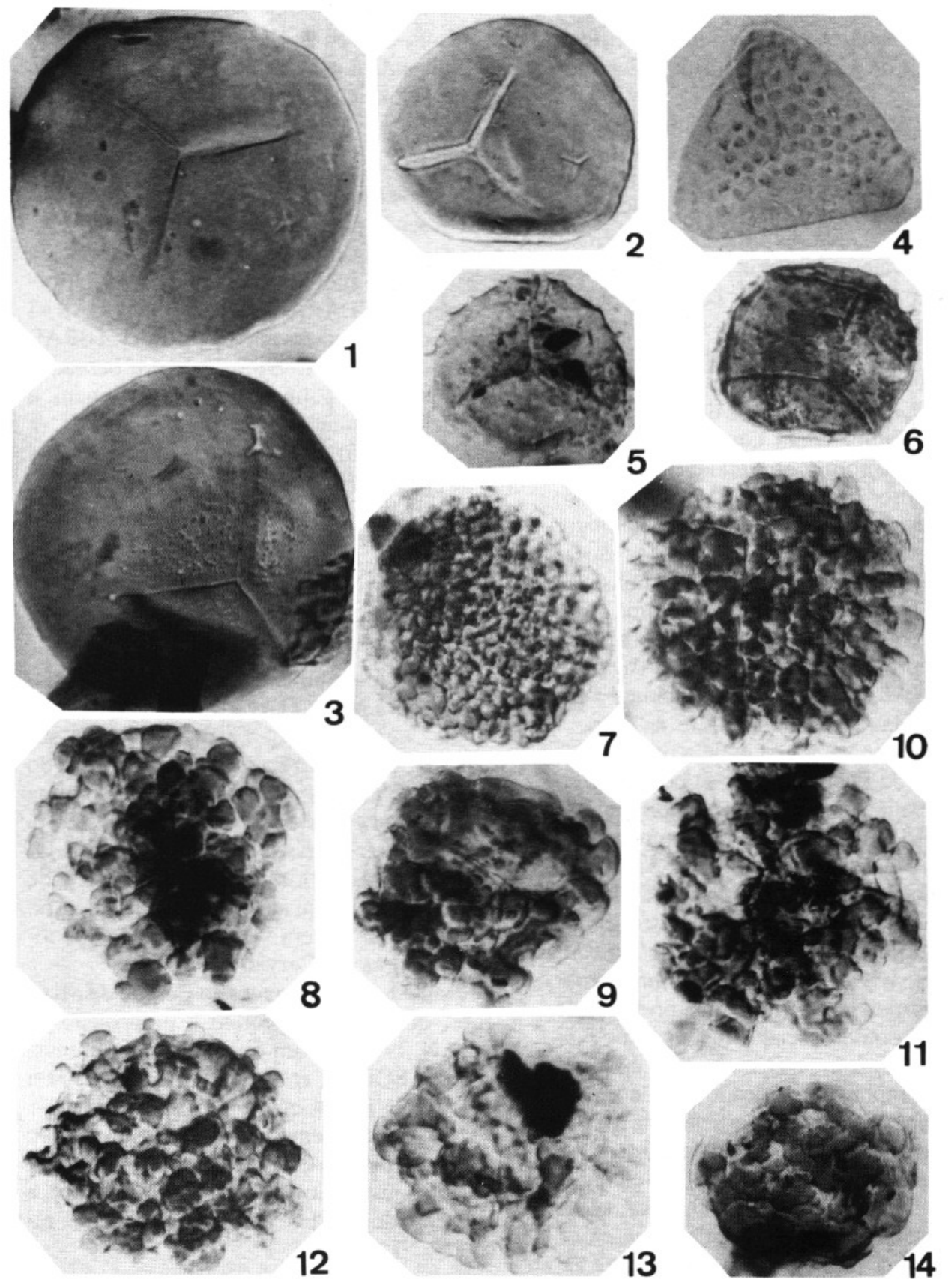

ESTAMPA I

Todas as figuras aumentadas 700 vezes.

Fig. 01 - Punctatisporites gretensis. Lâm. GP/4T-299.

Fig. 02 - Punctatisporites gretensis f. minor. Lâm. GP/4T-300.

Fig. 03 - Punctatisporites foveolatus. Lâm. GP/4T-302.

Fig. 04 - Granulatisporites sp. cf. G. varigranifer. Lâm. GP/4T-300.

Fig. 05 - Apiculiretusispora sp. cf. A. tuberculata. Lâm. GP/4T-301.

Fig. 06 - Apiculatisporis. sp. Lâm. GP/4T-304.

Fig. 07 - Convolutispora sp. Lâm. GP/4T-302.

Fig. 08 - Secarisporites sp. Lâm. GP/4T-301.

Fig. 09 - Secarisporites irregularis Lâm. GP/4T-300.

Fig. 10 - Raistrickia rotunda. Lâm. GP/4T-303.

Fig. 11 - Raistrickia sp. 1. Lâm. GP/4T-301.

Fig. 12 - Raistrickia rotunda. Lâm. GP/4T-299.

Fig. 13 - Raistrickia paganciana. Lâm. GP/4T-300.

Fig. 14 - Raistrickia cf. $R$. paganciana. Lâm. GP/4T-301. 

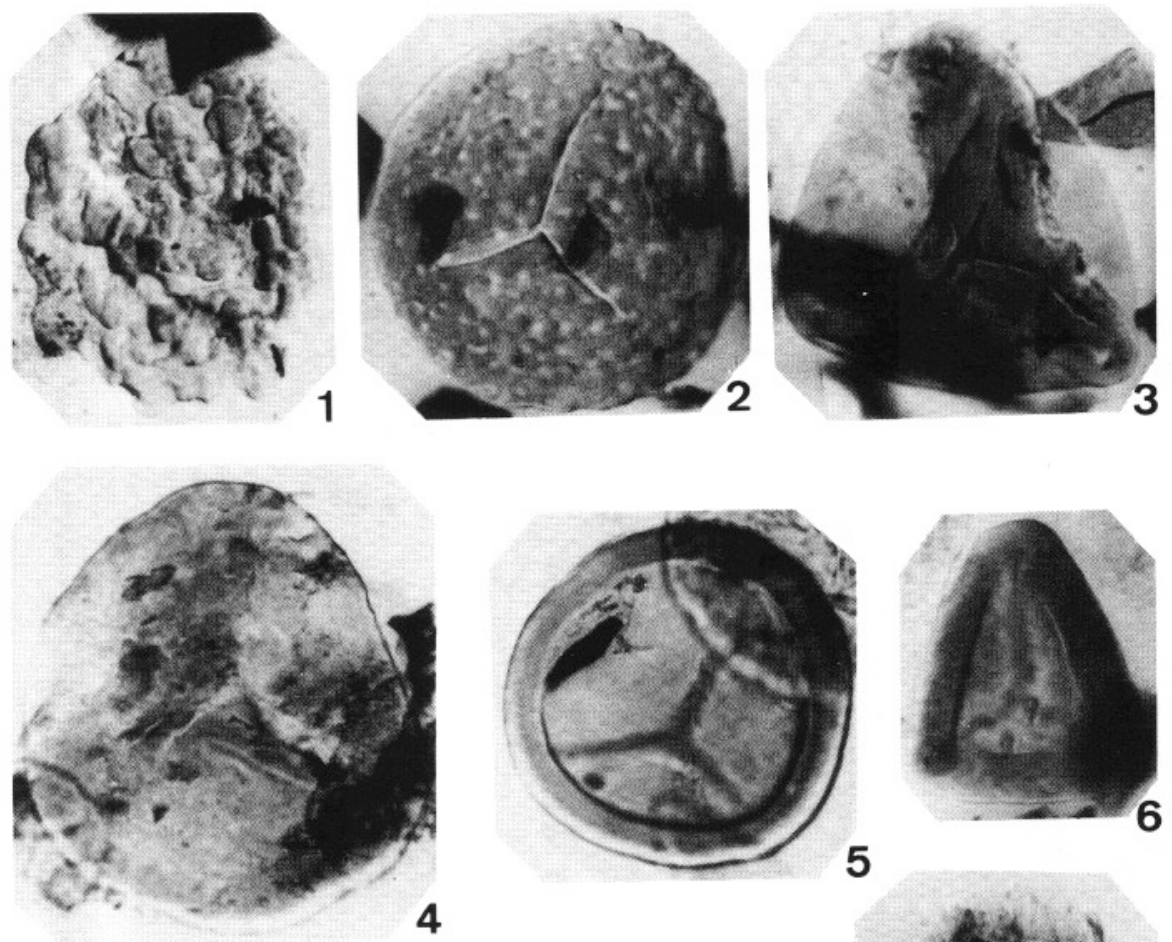

\section{4}
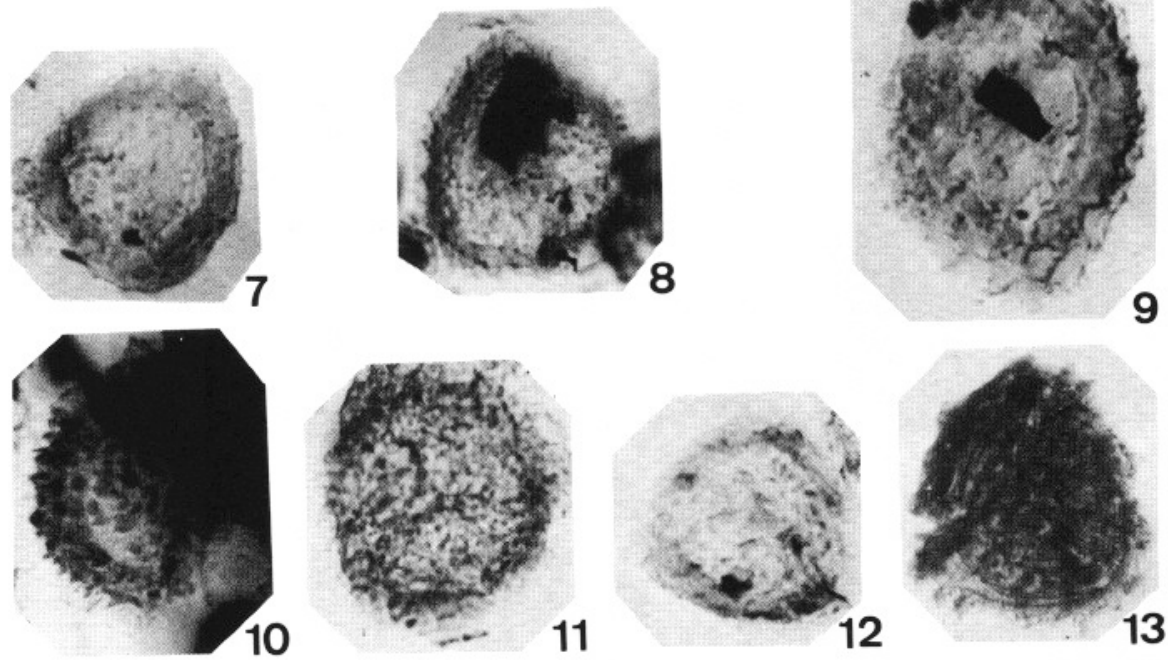

ESTAMPA 2

Todas as figuras aumentadas 700 vezes.

Fig. 01 - Raistrickia sp. 2. Lâm. GP/4T-302.

Fig. 02 - Foveosporites hortonensis. Lâm. GP/4T-299.

Fig. 03 - Dictyophyllidites sp. 1. Lâm. GP/4T-303.

Fig. 04 - Dictyophyllidites sp 2. Lâm. GP/4T-300.

Fig. 05 - Stenozonotriletes clarus. Lâm. GP/4T-301.

Fig. 06 - Murospora bicingulata. Lâm. GP/4T-299.

Fig. 07 - Densosporites sp. Lâm. GP/4T-301.

Fig. $08-$ Cristatisporites $\mathrm{sp} .1$

(Ancistrospora sp.). Lâm. GP/4T-302.

Fig. $09-$ Cristatisporites inordinatus.

(=Ancistrospora inordinata). Lâm. GP/4T-302.

Fig. $10-$ Cristatisporites of. C. spinosus.

(=Ancistrospora cf. A. spinosa). Lâm. GP/4T-303.

Fig. 11 - Cristatisporites spinosus

(=Ancistrospora spinosa). Lâm. GP/4T-302.

Fig. 12 - Cristatisporites menendezii

(=Ancistrospora cf $A$. verrucosa). Lâm. GP/4T-301.

Fig. 13 - Cristatisporites sp. Lâm. GP/4T-306. 

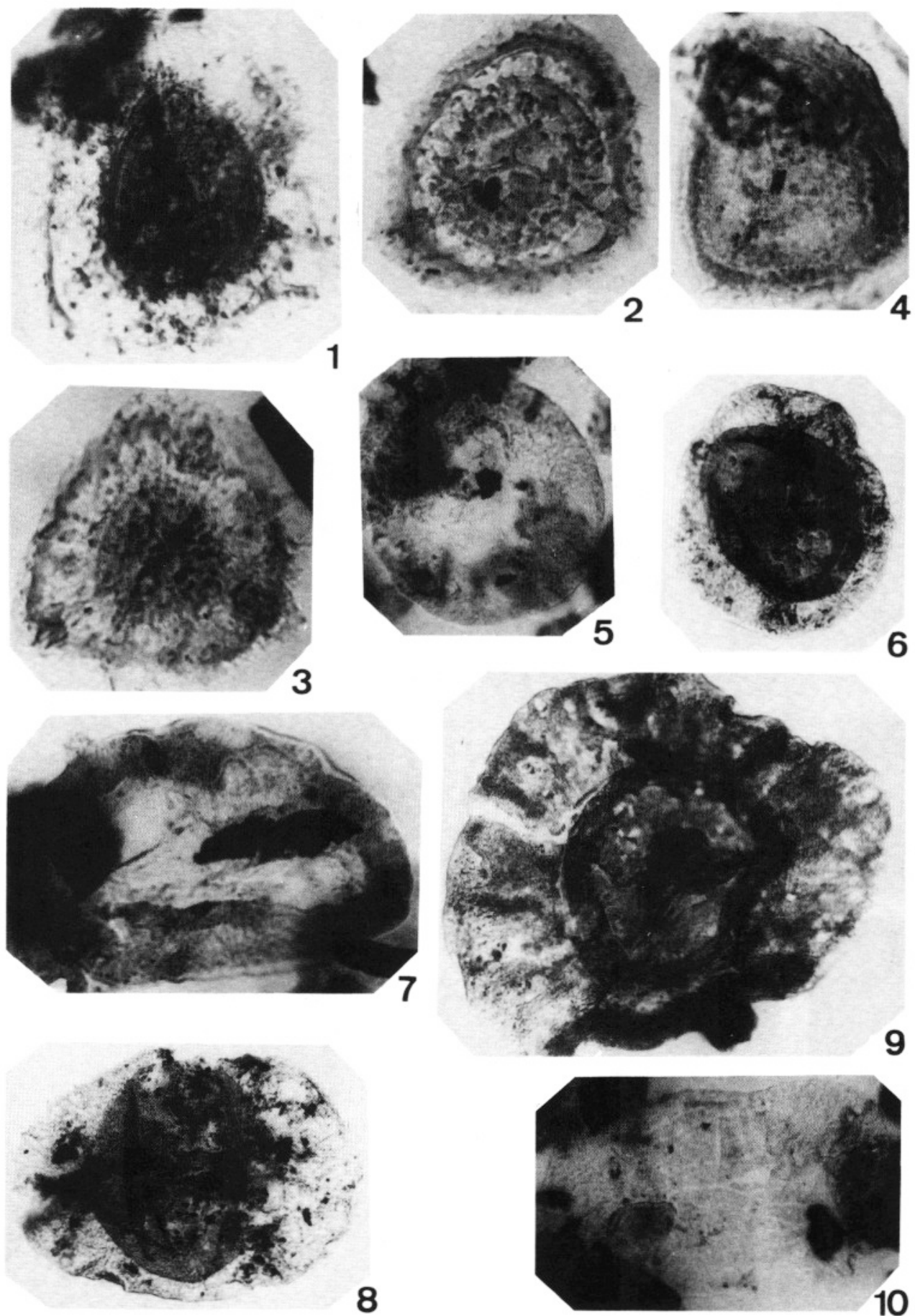

ESIAMPA

Figuras 1 a 4 aumentadas 700 v心

Figuras 5 a 10 aumentadas 350 veces.

Fig. 01 - Vallatisporites sp. cf. V c ciliaris. Lâm. GP/4T-305.

Fig. 02 - Vallatisporites vallatus Lâm. GP/4T-299.

Fig. 03 - Vallatisporites spinosus Lâm. GP/4T-299

Fig. 04 - Lundbladispora brazilie'nsis. Lam. GP/4T-303.

Fig. 05 - Florinites sp. Lâm. GP/4T-303.

Fig. 06 - Plicatipollenites indicus. Lâm. GP/4T-306.

Fig. 07 - Divarisaccus sp. Lâm. GP/4T-300.

Fig. 08 - Potonieisporites hrasiliensis. Lâm. GP/4T-305.

Fig. 09 - Plicatipollenite's trigonalis. Lâm. (iP/4T-306.

Fig. 10 - Protohaploxypinus sp. Lâm. GP/4T-299. 
mentos e intromissões de diques e sills de diabásio nos sedimentos paleozóicos da Bacia do $\mathrm{Pa}$ raná. Como resultado, em alguns casos deslocamentos verticais são tão significativos a ponto de originar falsas interpretações estratigráficas.

Seja por um motivo, seja pelo outro, os depósitos de Carvão de Buri têm sido posicionados, até o presente, na parte média (e.g. SAAD, 1977; MILLAN, 1987), médio-superior (RÖSLER \& PERINOTTO, 1985), ou então superior (e.g. CABRAL JÚNIOR \& MOTTA, 1985) do Subgrupo Itararé no Estado de São Paulo. No entanto, o resultado palinológico ora obtido mostra que o intervalo analisado corresponde cronologicamente à parte basal aflorante desta unidade, na medida em que outros níveis estratigráficos do Itararé têm revelado idades bem mais novas. Como exemplo cita-se o ritmito de Itu (DINO et al., 1987), datado no Permiano e correspondendo ao subintervalo $\mathrm{I}_{1}$ do esquema de DAEMON \& QUADROS (1970).

Cronoestratigraficamente, os depósitos costeiros de Buri correlacionam-se, grosso modo, aos sedimentos marinhos de Araçoiaba da Serra e parecem corresponder às primeiras ingressões marinhas detectadas na seqüência permocarbonífera da Bacia do Paraná. Caso isto seja verdadeiro, tudo leva a crer que esta ingressão corresponda ao primeiro ciclo deposicional marinho, registrado em subsuperfície por CAMPANHA et al. (1989), cuja zona de condensação foi chamada de "Folhelho Roncador". Tal seqüência foi registrada no poço 1-RO-1-PR (Roncador $\mathrm{n}$. 1), no intervalo $3.586-3.633 \mathrm{~m}$ de profundidade.

Dentro dessa concepção, o modelo de sedimentação proposto por CAETANO-CHANG \& LANDIM (1987) para o Subgrupo Itararé, na área de estudo, torna-se bastante plausível. Ele é composto por um trato de sistemas que inclui leques aluviais e rios entrelaçados oriundos de regiões altas glaciais, gradando para um sistema deltaico e deste para um ambiente marinho raso, sob a influência de um suprimento glacial.

Ressalta-se aqui, entretanto, que tal cenário é válido apenas para a parte basal do Subgrupo Itararé na região sul do território paulista, tendo em vista não existirem elementos seguros para estender este modelo para outras regiões do Estado.

Fora do Brasil, a única possibilidade de correlação é com sedimentos da Bacia de Paganzo (Argentina) já que esta parte da seção está aparentemente ausente em outros blocos gondvânicos, aí incluindo-se África, Índia, Austrália e Antártida. Na referida Bacia, onde conforme visto foram originalmente descritos níveis equivalentes palinologicamente enquadrados na Palinozona mais primitiva de AZCUY (1976) ou de AZCUY \& JELIN (1980), os resultados cronoló- gicos sugerem que a correlação, a nível litoestratigráfico, deve ser efetuada com o Membro Estratos Carbonosos da Formação Malanzán.

\subsection{Paleoecologia}

Conforme já referido, com base no empilhamento sedimentar e na faciologia presente, CABRAL JÚNIOR \& MOTTA (1985) concluíram que os depósitos de carvão de Buri teriam sido formados por baixios situados em uma planície deltaica, a qual estaria associada a um mar raso plataformal.

O conjunto microflorístico ora identificado - onde predominam elementos tipicamente continentais constituídos por licófitas (representadas por Densosporites, Ancistrospora, Cristatisporites, Vallatisporites e Lundbadispora), esfenófitas (Laevigatosporites) e filicófitas (Punctatisporites, Raistrickia, Granulatisporites e Apiculatisporites), sobre formas microplanctônicas marinhas (Michrystridium) - suporta, a princípio, a interpretação paleoambiental postulada por CIANTELLI JÚNIOR et al. (1983) e CABRAL JÚNIOR \& MOTTA (1985). Tal assertiva é válida, por um lado, porque os dados disponíveis denotam significativo suprimento terrígeno à bacia de sedimentação marinha, e por outro mostram que a vegetação presente é característica de zonas baixas e úmidas, de acordo com conclusões divulgadas por AZCUY (1975c) para um conjunto microflorístico correlato.

A influência marinha na planície de inundação é indicada pela presença do gênero Michrystridium, característico de águas rasas costeiras (WALL, 1965).

\section{PRINCIPAIS CONCLUSÕES}

A análise da composição microflorística do Carvão de Buri permite, em termos gerais, enumerar as seguintes conclusões:

- os níveis portadores de carvão da região de Buri registram uma diversificada palinoflora, cujo domínio é de esporos triletes lisos, zonados, murornados e cingulados;

- bioestratigraficamente, a palinoflora estudada se enquadra na Palinozona I de AZCUY (1976) e na Palinozona de Ancistrospora de AZCUY \& JELIN (1980), estabelecidas para sedimentos carboníferos da Bacia de Paganzo, Argentina. Na Bacia do Paraná, as assembléias que mais se assemelham são as de Monte Mor e Araçoiaba da Serra, porém é a de Buri, pela ancestralidade de seus elementos, a mais antiga;

- em termos litoestratigráficos, o Carvão de Buri correlaciona-se com a Formação Malanzán (Membro Estratos Carbonosos) da Bacia de Paganzo, Argentina; 
— uma idade westfaliana (Carbonífero Superior) é indicada para a associação, que se revela como a mais antiga assembléia de palinomorfos já descrita para o Neopaleozóico da Bacia do Paraná, contrariando o conceito pelo qual seria o Carvão de Monte Mor o mais antigo;

- o domínio de representantes de elementos continentais (licófitas, esfenófitas e filicófitas) sobre formas microplanctônicas marinhas (acritarcas), aliado às interpretações de caráter litofaciológico, conduz à reconstituição de um cenário paleogeográfico caracterizado por planície deltaica de um sistema deltaico aos níveis portadores do Carvão de Buri.

\section{AGRADECIMENTOS}

Os autores agradecem ao Geólogo Marsis Cabral Júnior e ao Prof. Paulo Roberto dos Santos pela cessão de amostras para estudo. Agradecimentos são também devidos à Prof ${ }^{\text {a }}$. Vilma Alves Campanha pelas críticas e sugestões, ao Prof. Dr. Thomas Rich Fairchild pela revisão crítica do Abstract e ao Prof. Dr. Jean Pierre Ybert pela cessão do equipamento fotográfico. Finalmente são aqui consignados agradecimentos à FAPESP pela cessão da bolsa de estudo ao autor sênior, que tornou possível a realização deste trabalho.

\section{REFERÊNCIAS BIBLIOGRÁFICAS}

ARAI, M. 1980. Contribuição dos polens estriados na bioestratigrafia do Neopaleozóico da parte NE da Bacia do Paraná. Bol. IG, USP, São Paulo, 11:125-135.

ARCHANGELSKY, S. \& GAMERRO, J. C. 1979. Palinologia del Paleozoico Superior en el subsuelo de la Cuenca Chacoparanaense, Republica Argentina. Parte I. Revista Española de Micropaleontologia, $11(3): 417-478$.

AZCUY, C. L. 1975 a. Miosporas del Namuriano y Westfaliano de la Comarca MalanzánLoma Larga, Provincia de La Rioja, Argentina. Parte I. Ameghiniana, 12(1):1-69.

AZCUY, C. L. 1975 b. Miosporas del Namuriano y Westfaliano de la Comarca MalanzánLoma Larga, Provincia de La Rioja, Argentina. Parte II. Ameghiniana, 12(2):113-163.

1975 c. Las associaciones palinologicas del Paleozoico Superior de Argentina y sus relaciones. In: CONGRESSO ARGENTINO DE PALEONTOLOGIA E BIOESTRATIGRAFIA, Tucumán, Atas, vol. 1 , p. 455-477.

1976. A review of the early Gondwana Palynology of Argentina and South America: a review. In: INTERNATIONAL PALYNOLOGICAL CONFERENCE, 4, Lucknow, Índia, vol. 2, p. 175-185.

AZCUY, C. L. \& JELIN, R. 1980. Las palinozonas do limite carbonico-permico en la Cuenca Paganzo. In: CONGRESSO ARGENTINO DE PALEONTOLOGIA E BIOESTRATIGRAFIA, 2, Atas, Buenos Aires, vol. 4, p. 51-67.

BOSE, M. N. \& KAR, R. K. 1966. Paleozoic sporae dispersae from Congo. I- KinduKalima and Walikale regions. Ann. Mus. Roy. Afr. Centr., Sér. In. 8a., Sc. Geol., 53:1-23.
BURJACK, M. I. A. 1978. Estudo palinológico da jazida carbonífera de Charqueadas. Dissertação de Mestrado, UFGo, 204 p., 7 est. (inédita).

CABRAL JÚNIOR, M. \& MOTTA, J. F. M. 1985. Geologia da Formação Itararé e sua potencialidade para carvão na região de Buri/Itapeva, SP. In: SIMPÓSIO REGIONAL DE GEOLOGIA, 5, São Paulo. Atas, vol. 2, p. 459-472.

CAETANO-CHANG, M. R. \& LANDIM, P. M. B. 1987. Evolução ambiental da sedimentação do Subgrupo Itararé na área de Itararé-Itaporanga (SP). In: SIMPÓSIO REGIONAL DE GEOLOGIA, 6, Rio Claro. Atas, vol. 1, p. 339-354.

CAMPANHA, V. A.; SAAD, A. R.; GAMA JÚNIOR, E. \& PULEGHINI FILHO, P. 1989. A caracterização marinha do Grupo Itararé (Permocarbonífero), Bacia do Paraná. In: SIMPÓSIO DE GEOLOGIA DO SUDESTE, 1, Rio de Janeiro, Boletim de resumos, p. 5.

CAUDURO, A.D. 1970. Lower Gondwana miospores from São Sepé outcrop (Rio Grande do Sul, Brazil). Publicação Especial, Escola de Geologia UFRGS, Porto Alegre, vol. 17, p. 1-34.

CIANTELLI JÚNIOR, C.A.; CABRAL JÚNIOR, M. \& NAKANO, S. 1983. Avaliação preliminar das ocorrências de carvão mineral na região de Buri-Itapeva, SP. In: SIMPÓSIO REGIONAL DE GEOLOGIA, 4, São Paulo. Atas, p. 353-366.

DAEMON, R.F. 1974. Integração dos dados palinológicos aos de fauna e flora das camadas fossilíferas da Bacia do Paraná - Implicações estratigráficas e paleogeográficas. Revista Unimar, 1:25-40.

\& QUADROS, L.P. 1970. Bioestratigrafia do Neopaleozóico da Bacia do Pa- 
raná. In: CONGRESSO BRASILEIRO DE GEOLOGIA, 24, Brasília, Anais, p. 359-412.

DINO, R.; LIMA, M.R. de \& ROCHACAMPOS, A.C. 1987. Palinologia do varvito (Subgrupo Itararé, Neopaleozóico) da região de Itu, São Paulo. In: CONGRESSO BRASILEIRO DE PALEONTOLOGIA, 10, Rio de Janeiro, resumo das comunicações, p. 46.

FABRICIO, M.E.D. 1981. Palinologia da Formação Rio Bonito na área de Gravataí-Morungava, Rio Grande do Sul. Pesquisas, 14:69-130.

FERREIRA, R.L. \& SOMMER, F.W. 1982. Megásporos paleozóicos do Estado de São Paulo. Anais da Academia Brasileira de Ciências, 54(4):721-728.

FOSTER, C.B. 1979. Permian plant microfossils of the Blair Athol Coal Measures, Baralaba Coal Measures, and basal Rewan Formation of Queensland. Geol. Surv. Queensl. Publ. 372, Palaeontol. Paper, 45:1-244.

HART, G.F. 1965. The systematics and distribution of permian miospores. Witwatersrand Univ. Press., Johannesburg, 252 p.

LIMA, M.R.; DINO, R. \& YOKOYA, N.S. 1983. Palinologia de concreções calcíferas do Subgrupo Itararé (Neopaleozóico da Bacia do Paraná) da região de Araçoiaba da Serra, Estado de São Paulo. Anais da Academia Brasileira de Ciências, 55(2): 195-208.

MAHESWARI, H.K. 1967. Studies in the Glossopteris flora of India: miospore assemblage from the Lower Gondwana Exposures along Bansloi river in Rajmahal hills, Bihar. Palaeobotanist, 15(3):258-280.

1969. Palaezoic sporae dispersae from Congo. X Microfossils from a cliff section at the confluence of Lufupa and Mushyasha rivers, South Katanga. Ann. Mus. Roy. Afrq. Centrale, Belgique, Sci. Geol., 63:113-168.

MARQUES-TOIGO, M. 1973. Estudo palinológico de concreções calcárias da Formação San Gregório, NE da República Oriental del Uruguai, Bacia do Paraná. Dissertação de Mestrado, UFRGS, 109 p. (inédita).

\& PONS, M.E. 1976. Estudo Palinológico do Furo de Sondagem P7 - Malha Oeste da Bacia Carbonífera de Irui, RS, Brasil. In: CONGRESSO BRASILEIRO DE GEOLOGIA, 28, Porto Alegre, Anais, p. 278-288.

MENENDEZ, C.A. \& AZCUY, C.L. 1972. Ancistrospora, un nuevo genero de miospora del Carbonico de La Argentina. Revista Es- pañola de Micropaleontologia, 4(2):157-168.

MILLAN, J.H. 1987. Os Pisos Florísticos do carvão do Subgrupo Itararé no Estado de São Paulo e suas implicações. In: CONGRESSO BRASILEIRO DE PALEONTOLOGIA, 10, Rio de Janeiro. Anais, p. 831-857.

OTTONE, E.G. \& AZCUY, C.L. 1990. Palinologia del carbon del Agua Hedionda (Carbonífero). Huaco. Provincia de San Juan, Argentina. Ameghiniana, 27(1-2):3-18.

PLAYFORD, G. 1978. Lower Carboniferous spores from the Ducabrook Formation, Drummond Basin, Queensland. Palaeontographica, Abt. B., 167:105-160.

PONS, M.E. 1975. Estudo palinológico do Subgrupo Itararé na “'Coluna White", Permiano Inferior, Santa Catarina. Dissertação de Mestrado, UFRGS, p. 1-104. (inédita).

RÖSLER, O. \& PERINOTTO, J.A.J. 1985. Nota sobre a ocorrência de Nothorhacoptcris sp. em siltitos da porção médio-superior do Subgrupo Itararé em amostra de subsuperfície no município de Buri, Estado de São Paulo. Paleobotânica Latinoamericana, Circular Informativa da ALPP, 7(1):25.

SAAD, A.R. 1977. Estratigrafia do Subgrupo Itararé no Centro e Sul do Estado de São Paulo. Dissertação de Mestrado, IG-USP, 107 p., (inédita).

SOMMER, F.W. \& TRINDADE, N.M. 1965. A flora gondwânica brasileira à luz da pesquisa esporológica. In: CONGRESSO BRASILEIRO DE GEOLOGIA, 19, resumo das comunicações.

SOUZA, P.A. de; LIMA, M.R. de \& SAAD, A.R. 1993. Palinologia dos carvões paleozóicos do Estado de São Paulo. II - O Carvão de Monte Mor. Academia Brasileira de Ciências, 65(3):327.

SOUZA-FILHO, E.E. 1986. Mapeamento faciológico do Subgrupo Itararé na Quadrícula de Campinas (SP). Dissertação de Mestrado, IG-USP, 121 p., (inédita).

SUNDARAM, D. 1980. Observações palinológicas sobre alguns sedimentos do Gondwana Inferior da Bacia do Paraná, Brasil. Bol. $I G$, USP, 11:161-189.

1987. Palinologia do Subgrupo Itararé (Neopaleozóico), Bacia do Paraná, no Estado de São Paulo, Brasil. Tese de Doutoramento, IG-USP, 311 p., 10 est., (inédita).

TRINDADE, N.M. 1959. O gênero Lagenoisporites no Gondwana brasileiro. DNPM/ DGM, Notas preliminares e estudos, bol. 112:1-13, 2 est. 
TRINDADE, N.M. 1960. O gênero Trileites no Gondwana brasileiro. DNPM/DGM, Notas Preliminares e Estudos, bol. 114:1-17, 4 est.

1970. Megásporos carboníferos de Monte Mor, Estado de São Paulo. Anais da Academia Brasileira de Ciências, 42(3):459-470.

\& SOMMER, R.W. 1966. Sinopse da sistemática dos megásporos do Gondwana brasileiro. Anais da Academia Brasileira de Ciências, 38(2):305-314.

WALL, D. 1965. Microplankton, pollen and spores from the Lower Jurassic in Britain. Micropaleontology, 11:151-190.

YBERT, J.P. 1975. Etude des miospores du Bassin Houiller de Candiota-Hulha Negra, Rio Grande do Sul, Brésil. Pesquisas, 181-227.

Contribuição ao Projeto 237 "Floras of the Gondwanic continents". Trabalho apresentado na VII Reunião de Paleobotânicos e Palinólogos (São Paulo, 10-16/12/90).

Endereço dos autores:

- Paulo Alves de Souza - Instituto Geológico - SMA - Caixa Postal 8772 - 04301-903 - São Paulo, SP - Brasil.

- Murilo Rodolfo de Lima - Universidade de São Paulo - Instituto de Geociências - Caixa Postal 2089901498-970 - São Paulo, SP - Brasil.

- Antonio Roberto Saád - Divisão de Geologia e Recursos Minerais - Instituto de Pesquisas Tecnológicas do Estado de São Paulo - IPT - Cidade Universitária Armando Salles Oliveira - Butantã - Caixa Postal 7141 - 05508-901 - São Paulo, SP - Brasil. 